of the desired tritium incorporation far greater than that of the undesired side-reactions. In selecting the ratio of adsorbed substance to charcoal carrier, one must consider that at a low concentration of the substance to be tritiated on charcoal a finer distribution on the carrier can be achieved with consequently a higher rate of tritium incorporation. However, at still lower substance to charcoal ratios a high percentage of the tritium radiation energy will contact only the charcoal and initiate no exchange reaction. Therefore, there must be an optimal relation between the amount or adsorbant and adsorbed substance as described by Meshi et al. ${ }^{5}$. The lower specific activities obtained by these workers when mechanical mixtures of charcoal and organic compounds were exposed to tritium gas as compared to exposure of the pure compound are understandable.

From our results so far obtained we are encouraged to continue our studies of the critical factors in the Wilzbach tritium gas exposure technique with the view of increasing the specific activities readily attainable, particularly for natural products for which other labelling methods are not available.

Rainer Maurer Martin Wenzel.

Peter Karlson

Physiologisch-Chemisches Institut,

Freie Universität Berlin

(Berlin 33), and

Physiologisch-Chemisches Institut,

Universität München

(München 15), Germany.

${ }^{1}$ Wilzbach, K. E., J. Amer. Chem. Soc., 79, 1013 (1957).

'Wenzel, M., and Schulze, P. E., Tritiummarkierung (Walter De Gruyter and Co., Berlin, 1962).

"Wenzel, M., Wollenberg, H., and Schulze, P. E., Tritium in the Physical and Biological Sciences, I.A.B.A., 2, 37 (Vienna, 1962). Wollenberg. H., and Wenzel, M., Z. Naturforschung, 18, b, 8 (1963).

- Karlsor, P., Maurer, R., and Wenzel, M., Z. Naturforschung, 18, b, 219 (1963).

s Meshi, T., and Takahashi, T., Bull. Chem. Soc. (Japan), 35, 1510 (1962).

- Tschesche, R., Wulff, G., and Balle, G., Tetrahedron, 18, 959 (1962)

'Wenzel, M., Wollenberg, H., and Schulze, P. E., Atompraxis, 7, 89 (1961).

${ }^{8}$ Walens, H. A., Turner, A., and Wall, M. E., Anal. Chem., 26, 325 (1954).

- Schulze, P. E., and Wenzel, M., Angew. Chem., 74, 777 (1962).

\section{Acid-catalysed Darzens Condensation of the Nitro-hydroxy-phenacylbromides}

IN a previous communication it was reported that the Darzens condensation of nitro-hydroxy-phenacylbromides and 4-nitro-benzaldehyde was successfully carried out not only in alkaline medium but also in ethanol saturated with hydrochloric acid ${ }^{1,2}$.

As the mechanism of Darzens condensation essentially resembles the reaction mechanism of chalcon formation and aldol condensation ${ }^{3,4}$, it may be assumed that the Darzens condensation occurring on the catalysing effect of hydrochloric acid takes place analogously to aldol condensation catalysed by acid, that is it can be described as follows :

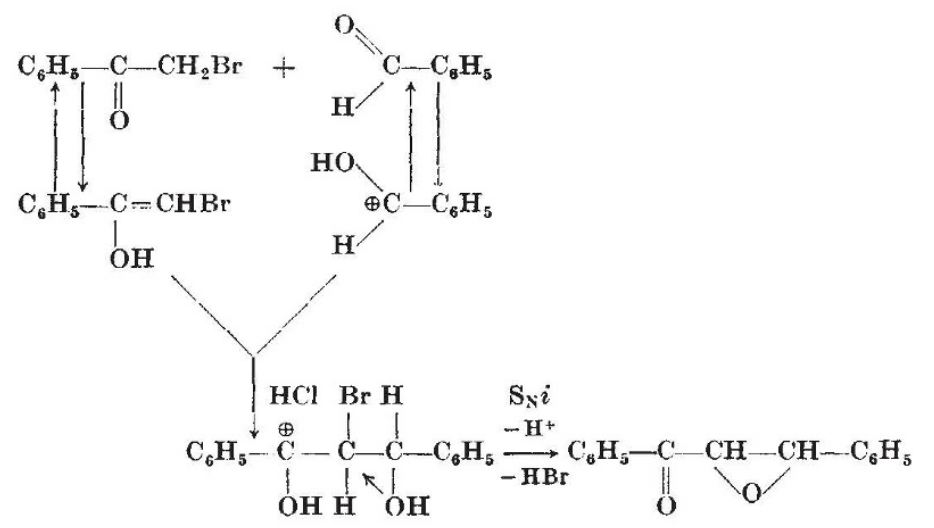

For the time being it was possible to carry out the Darzens reaction in hydrochloric acid medium only with benzaldehydes containing substituents of electron attraction (nitro and chlorine group) and with nitro-hydroxyphenacylbromides. Epoxy-ketone could not be isolated in the presence of aluminium chloride as catalyst. A.ll this indicates that the successful condensations likely represent Darzens reactions of anomalous run, but to establish this decisively further investigations are required.

The successful Darzens condensation in hydrochloric acid medium can be probably interpreted by the fact that the formed epoxy-ketones are stable even in strongly acid media. It is likely that the cause of stability of epoxyketones is the strong electron affecting substituents of the aromatic rings, owing to the electron-affecting effect of which, on one hand, carbonic atoms of the epoxy ring are relatively poor in electrons, and, on the other hand, the nucleophility of the oxygen of epoxy ring decreases, thus the ring can, relatively, scarcely be opened by acid, as, for example, in case of the following compound:<smiles>O=C(C1CCC(O)C([N+](=O)[O-])C1)C1OC1C1CCC([N+](=O)[O-])CC1</smiles>

The foregoing considerations on the effect of substituents are supported by other results ${ }^{5,6}$.

Gy. Sipos F. Sirokmáx

Institute of A.pplied Chemistry and Central Isotope Laboratory, University of Szeged, Hungary.

${ }^{2}$ Sipos, Gy., and Szabo, R., Acta Phys. Chem. (Szeged), 8, 48 (1962). ${ }^{2}$ Sipos, Gy., Czukor, B., -and Dob6, I., A.ta Phys. Chem. (Szeged), 9, 48 (1963).

${ }^{3}$ Ballester, M., and Bartlett, P. D., J. Amer. Chem. Soc., 75, 2042 (1953). 4 Ballester, M., and Perez-Blanco, D., J. Org. Chem., 23, 652 (1958).

s Elderfield, R. C., Heterocyclic Compounds, 1, 39 (1950). Winstein, S., and thylene Oxides, 1. - Parker, R. E., and Isaacs, N. S., Chem. Rev., 59, 755 (1959).

\section{Complex Formation with Trivalent Iron and $N, N$-Ethylene bis[2(o-hydroxyphenyl)] Glycine}

$N, N^{\prime}$-Ethylene bis [2(o-hydroxyphenyl)] glycine (EHPG) is one of the analogues of ethylenediamine tetraacetic acid (EDTA.) which has aroused increasing interest recently as a reagent forming very stable chelates with many metal ions. The ferric complex is particularly valuable for treating iron chlorosis in plants, as the use of the corresponding EDTA chelate is restricted to crops grown on acid and only moderately weak alkaline soils, but the ferric-EHPG complex is effective in correcting the mineral deficiency in plants grown on alkaline soils. Moreover, use of the ferric chelate has become important in analytical chemistry as a sensitive reagent for iron ${ }^{1,2}$. The compound has a red colour suitable for spectrophotometric determinations.

The stability constant of the ferric complex has previously been reported to be $>30$ (logarithmic) $^{3}$. As the stability is very high, the potentiometric tritrations did not allow exact determination. In our laboratory we are conducting polarographic investigations of polyamine-polycarboxylic acid chelates, among them the ferric and ferrous complexes of EHPG. The results of the investigations will be published elsewhere. Direct polarographic determinations do not allow calculations of the ferric stability constant. As we need it in the polarographic investigation, the constant is now evaluated separately by means of a spectrophotometric method. 